\title{
Personal ecologies of calendar artifacts
}

\author{
Anke Dittmar ${ }^{*}$ and Laura Dardar
}

\begin{abstract}
The use of calendars for work and personal activities has been widely investigated for decades and the term calendar work, coined by Palen (CHI 17-24,1999), refers to the many ways people employ and interact with calendars. Previous research has focused on calendar usage in specific domains or on the differences between paper and digital calendars. The current paper is positioned somewhat differently by exploring calendars as object in personal ecologies of calendar artifacts. In such personal calendar ecologies, the users, their tasks, their practices, and the calendar artifacts adapt and evolve together. In addition, individual users are typically engaged in various activities in specific contexts (realms) that are established and maintained by groups of people, supporting the overarching culture of these realms. As such, the web of common practices, activities and tasks, as well as the calendar artifacts shape the individual calendar work. To our knowledge, this article is the first study that investigates diverse personal ecologies of calendar artifacts. To this end we collected detailed user data with (a) exploratory interviews and (b) the Day-Reconstruction Method. The results indicate that the changing demands in daily life, the availability of new tools, and the participants' knowledge about the costs and benefits of their calendar work and about the consequences of potential failures influence their tendency to explore and possibly integrate new calendar artifacts and appear implicated in the deliberate non-use of new technology. It appears that paper and digital calendar artifacts continue to co-exist. The results indicate an existing 'appointment culture' with a high demand of precisely scheduled episodes, and the importance of calendar artifacts for maintaining work and personal relationships in the light of the travel and new technologies for communication.
\end{abstract}

Keywords: calendars; calendar work; personal artifact ecologies; appropriation; Day-Reconstruction Method

\section{Introduction}

Calendars have been used for thousands of years. They help humans to orient their bodies and minds in time and space, and therefore Leshed and Sengers [1] refer to them as technologies of the self. While early calendars followed the moon cycles and may have served for crops or family planning, the artifacts studied in this paper are based on the Gregorian calendar and support a perception of time in terms of days, weeks, months, and years. In the field of human-computer interaction (HCI), calendar use has been investigated for decades. Early work examined paper artifacts to inform the design of digital calendars [2-4]. In the 1990s, the use of electronic group calendars and mobile calendars were first explored in various work domains [5-8], and later also in domestic environments $[9,10]$. The literature review for this research also shows that many existing studies concentrate on specific

\footnotetext{
*Correspondence: anke.dittmar@uni-rostock.de

University of Rostock, Rostock, Germany
}

technologies and assume a comparison between paper and digital calendar tools.

The study presented in this paper applies the concept of artifact ecology to understand calendar practices of individual users in the current western world. The concept of artifact ecology developed by Kirsh [11] and others puts artifact use in a broader context by emphasizing the coadaptation and co-evolution of tasks, practices, artifact collections and users. The study was conducted in two parts: part 1 was an exploratory interview study and part 2 was a follow-up study using the Day-Reconstruction Method [12]. Interviews with thirteen students and five professionals do not indicate that the students prefer modern digital artifacts and older people rely on paper. Moreover, it appears that the dynamics in calendar use cannot be entirely understood by looking solely at individuals in certain roles (e.g., managers [7], mothers [10, 13], academics [14], students [15]) or at specific groups (e.g., institutions [6], companies [5], families [10]), but that it has to be analyzed in a wider context. Individuals in the

\section{Springer}

(c) 2015 Dittmar and Dardar. This is an Open Access article distributed under the terms of the Creative Commons Attribution License (http://creativecommons.org/licenses/by/4.0), which permits unrestricted use, distribution, and reproduction in any medium, provided the original work is properly credited. 
western culture are typically faced with different activity systems or realms that are established and maintained by specific groups of people (e.g., families, companies, friends, sport clubs), supporting the overarching culture of these realms. Each of those worlds has its own rhythms, needs, tasks and roles, values, and power relations. Calendar artifacts may partly reveal how individuals deal with their partly conflicting demands. While their behavior is shaped by dominant cultural values and experienced sub-cultures, their specific moves within and between different activities also contribute to reshape a culture in the small and in the large.

These aspects are taken into consideration in the second part of the present study. Three research questions guided this study: 1 . Which forms of personal ecologies of calendar artifacts can currently be observed? 2 . How have these ecologies evolved? For example, what factors have influenced the selection and introduction of specific calendar artifacts? 3. What are the possible net effects of personal calendar artifact ecologies? The Day-Reconstruction Method was adapted to analyze calendar work of six participants who span a loose network through partly overlapping activities and have longtime experience in using calendar artifacts to manage multiple and evolving activities. The study reveals the tight interplay between calendar and communication tools, human memory and trigger mechanisms in the diverse calendar practices of the participants. The results indicate that the changing demands in daily life, the availability of new tools, and the participants' knowledge about the costs and benefits of their calendar work and about the consequences of potential failures influence their tendency to explore and possibly integrate new calendar artifacts and appear implicated in the deliberate non-use of new technology. Explanations for appropriation processes and their effects on personal and more global calendar ecologies are provided. The results indicate an existing 'appointment culture' with a high demand of precisely scheduled episodes, and the importance of calendar artifacts for maintaining work and personal relationships in the light of the travel and new technologies for communication.

\section{Background and literature review}

What do I/we want to do, with whom, where, when, for how long, and with what means? Calendar artifacts help to form, manage, and realize intentions by supporting prospective remembering. Payne [4] points out that people deliberately rehearse intentions ("What do I need to do today?") or inadvertently recall them ("That reminds me, I must...") while browsing calendars.

Paper and digital calendars with their overviews of years, months, weeks, days, and/or hours reflect a culturally constituted temporal orientation, and thus provide a particularly effective support for appointments and scheduling. As shown in Fig. 1(a), many weekly views have less space for Saturdays and Sundays indicating that weekends typically need less scheduling in our culture [16]. Other temporal rhythms at personal, group or cultural level are only visible with calendar use. For example, all exemplars in Fig. 1 have very few, or no entries for the end of December.

In addition to temporal orientation, scheduling and reminding, calendars are also employed for note recording/archiving and later retrieval (e.g., meeting notes), and for tracking events (e.g., spending habits). Fig. 1(b) shows a tracking method: crossed out entries. Palen [5] coined the term calendar work for the above mentioned actions.

\section{Paper versus Digital Calendars}

Blandford and Green [6] point out that many calendar studies aim at identifying limitations of current artifacts to inform design processes of digital calendar tools (e.g., $[2-5,8,10])$. As a consequence, paper and digital calendars are often considered as competitive artifacts and their properties are compared to favor one over the other. For example, free-form annotation, opportunistic rehearsal [14], personalization, and emotional aspects [15] are seen as possible reasons for the continued use of paper that is reported in $[1,6,14,15]$. However, Kelley and Chapanis [2] noted that there is already a wide spectrum of paperbased calendars - 150 unique published formats alone have been mentioned. There are specific appointment calendars, journal calendars, calendars in different sizes and with different 'extras' etc. Kirsh [11] uses the term speciation to refer to structurally different artifacts that share a family resemblance but "are not direct competitors vying for supremacy in a single niche" because they are designed to meet the different needs of users. Later in this article, we suggest co-existing species of paper and digital calendar artifacts.

Studies about calendar use show that many individuals currently use a 'battery of tools as an ensemble' [6] and not an all-in-one tool (e.g., $[1,6,13,14])$. Below, frequently discussed aspects of calendar artifacts and observed usage practices are considered in more detail.

\section{Personal and shared calendars}

Crabtree et al. [9] understand calendars as "personal and social objects, providing a means for individuals to organize their affairs and a visible resource making others aware of the individual's schedule". They emphasize the fact that calendars can be shared to support social coordination and awareness. While paper calendars such as those in Fig. 1 are typically seen as personal objects (e.g., Tomitsch et al. [15]), wall calendars in domestic environments and digital group calendar systems (GCS) are examples of shared calendars. GCSs allow individuals, groups, and non-person entities (e.g. rooms or cars) to 


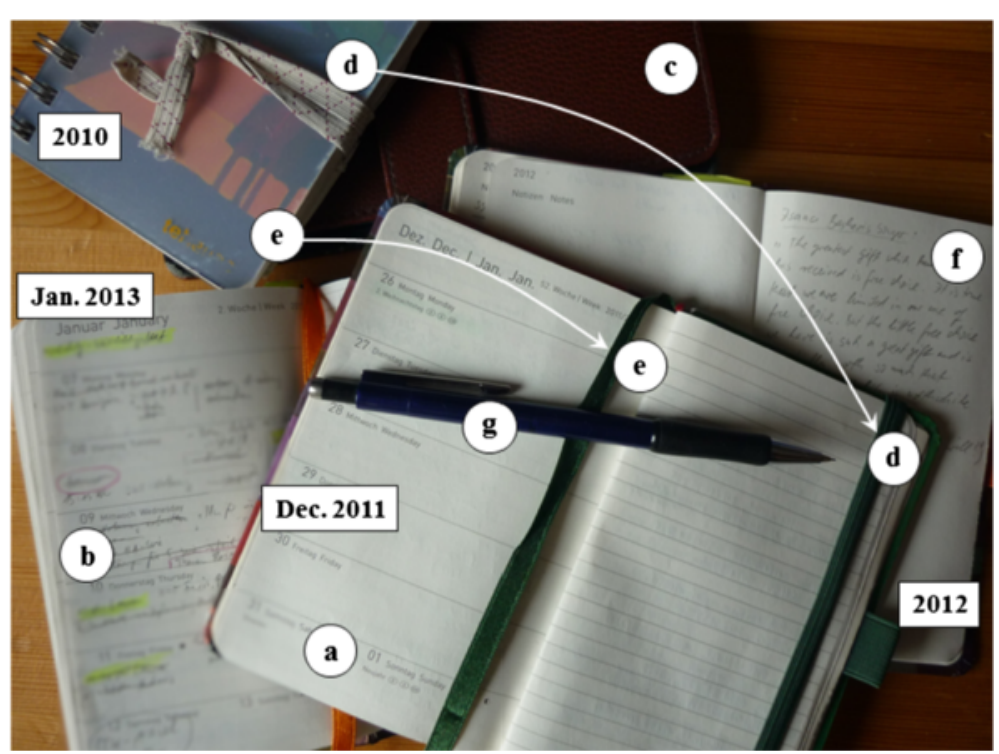

Fig. 1 Many people keep their calendars over years [2] like this participant in part 2 of the current study

keep their own calendar on-line and to define various degrees of access to other users [5]. They support meeting arrangements by an increased ability to reach other members of the group (connectivity) and by providing more information on time, location, and activities of collaborators (communality) [17]. Many authors who studied the use of GCSs have found that their success does not only depend on the functionality of the tool but also on the tasks and the characteristics of the users (e.g., [5, 17, 18]). Hooff [17] points out that "it is important to stimulate members of the organization to use their calendars in a 'collective' fashion and to establish organization-wide use" to exploit the benefits of electronic calendaring and scheduling.

\section{Portable and stationary calendars}

Like other work, calendar work is situated. >From a technical perspective, the accessibility and portability of calendar artifacts increases their likelihood to be ready-to-hand when needed. Mobile digital calendars are described by Sell and Walden [8] as a distinct category of calendar tools because they "combine the technological benefits of PC-based calendars with the portability benefit of paperbased calendars". Salzman and Palen [19] conducted an ethnographically-informed survey among employees of a large high-tech company. They noticed a tight coupling between GCSs and mobile calendars and paper calendars respectively which added mobility to the GCSs. They also found out that the percentage of mobile calendar users who keep just one calendar is higher than for any of the other calendar types and suggested that mobile calendar users manage their time more satisfactorily.
It should be noted though that people deliberately make use of location in their calendar work and, therefore, portable calendars are not better as such than stationary ones. A well-studied example is family wall calendars which are often located in the kitchen [9, $10,13]$ because it is an activity center in domestic spaces [20]. Stationary calendars also play an important role in supporting boundary work as described below.

\section{Realm-specific and multipurpose calendars}

Salzman and Palen [19] suggest that "there appear to be two distinct user populations: those who keep just one calendar (...no matter the calendar type), and those who more intensively keep multiple calendars (almost 2.5 calendars on average...)". The authors found out that job role seems to affect the type, but not the number, of calendars people use. Brush and Turner [21] report from their survey, which was also conducted in a high-tech company, that the participants used a median of three different calendars (paper and digital combined) to manage work and personal scheduling.

Nippert-Eng [22] is one of the few researchers who tries to analyze possible reasons for a person's use of one or of multiple calendars. She points out that, in our culture, people view themselves as having multifaceted identities which are related to specific places, times, and groups of people. Nippert-Eng [22] particularly considers 'home' and 'work' and the boundary work surrounding these realms of life. Boundary work is defined as "the process of creating and maintaining more or less distinct 'territories of the self"' and it is suggested that it can be 
described by an integration-segmentation continuum. For the extreme integrator, all space and time is multipurpose and there exists no distinction between what belongs to 'home' and 'work'. In contrast, 'home' and 'work' are exclusive categories for the extreme segmentor. There is no conceptual, physical or temporal overlap between them. According to Nippert-Eng [22], integrators are supported by integrating strategies of calendar maintenance, for example by one portable calendar tool or by listing the same information on multiple calendars. Segmentors typically have realm-specific calendars both in location and content.

\section{Functionality, usability, user experience}

Figure 1 shows three types of mass produced pocket calendars that a person has used over a period of years. Although the owner was pleased with the functionality of the exemplar marked by (c) it has been replaced in 2010 by a smaller and more lightweight one which was easier to carry around. Missing functionality was added to the new calendar: a rubber strap to hold additional paper notes (Fig. 1(d)). The owner also improved its usability by cutting edges to access the current week more easily (Fig. 1(e)). The type of calendar used from 2011 to 2013 already has a rubber strap and a paper pocket serving (d) as well as a ribbon serving (e). It also contains some blank pages which support the user's habit of spontaneously writing down quotes she encounters (Fig. 1(f): "The greatest gift which humanity has received is free choice. It is true that we are limited in our use of free choice. But the little free choice we have is such a great gift and it is potentially worth so much that for this itself life is worthwhile living." - Isaac B. Singer). Personal calendars are emotional and very intimate artifacts [15] which need to be designed for a good user experience.

As described earlier in this section and in the introduction, the objective of most calendar studies has been to inform design by eliciting functional requirements and by identifying usability problems of existing systems. Just to give one more example, Starner et al. [23] asked their study participants to specify their primary calendar system used away from desk. In a second part of the study, participants (most of them male university students) were invited to a face-to-face appointment scheduling conversation. The results show that a significant number of the participants switched to temporary calendar devices to buffer short-term appointment information for later reconciliation with their primary device. The authors suggest that the applied temporary device was more usable in terms of navigation time and less disruptive to the conversation than the claimed primary device. This and other studies suggest that the use of multiple calendar artifacts can also be a result of functional or usability deficiencies of single artifacts.

\section{Calendar artifact collections}

Calendar artifact collections refer, in the context of this article, to an individual's or a group's actual set of calendar artifacts including paper notes, to-do lists, schedules, and reminders such as alerts, physical objects, and other people $[1,4,6,14,24]$. While paper and digital calendars especially support a temporal orientation other tools focus on other aspects in intention forming and management. A shopping list, for example, can be created collaboratively [24] to provide information of what is needed. However, nothing is made explicit about when the items of the list are to be bought and by whom. This might not have been decided yet or it might be routine and the person will remember their shopping task.

Furthermore, studies have found that people prioritize between some of their calendar tools (e.g., primary and secondary calendars are mentioned by Salzman and Palen [19]). Palen [5] refers to the risks of competing calendar artifacts. For heavy calendar users, the "maintenance of multiple artifacts is rarely without some struggle, and often one calendar artifact loses the battle".

Alternative terms for calendar artifact collections that are used in the literature are time management tools [6], productivity tools [1], and informational artifacts which form organizing systems in the home [13].

Calendars work in combination with internal resources and with other external tools. The small calendars in Fig. 1 fit the needs of the owner because she uses a pencil with a very thin lead (Fig. 1(g)). Appointments and collaborative scheduling require that collaborators 'obey' synchronized clocks and that shared communication tools exist. Although people do not need to watch the time if they use electronic reminders some internal resources are still necessary to actually trigger the intended action. Generally, there is a blurred boundary between calendar artifacts and communication tools. Many people also depend on email for their time and task management [19] and most GCSs have integrated email applications.

\section{Calendar practices in specific domains}

According to Zerubavel [25], time is increasingly measured and treated as a commodity in the western world. For a better understanding of existing perspectives on time, it can be useful to consider 'extreme' forms of time management practices in specific domains. In hospitals, and in particular in perioperative systems, as one such well-studied domain, coordination is a deeply collaborative process. Some members of the staff are exclusively concerned with coordination tasks to ensure a proper treatment of all patients with maximum utilization of operating rooms which are the most costly hospital facilities [26]. Bardram [27] shows that coordination work is supported by tools at three temporal levels: allocation, scheduling, and synchronization. Allocation is the 
long-term agreement on the usage of shared resources and often has a rhythmic structure to support its memorization and use by the collaborators (e.g., a working meeting every Monday morning). Allocation is important for constraining the creation of temporal plans (scheduling), which in turn prepare fine-grained temporal coordination (synchronization) of the actual events in an operation room suite. Although time management strategies in other domains or realms of life may be less refined and detailed, and perhaps less visible, they also reveal the above mentioned temporal levels as we will discuss later.

\section{Study}

The current study consisted of two parts. The first part was set of exploratory interviews conducted with students and professionals between October and December 2012. The objectives were to collect data on current calendar practices, and relate these observations to previous work, and inform part 2 of the study. While most reported calendar studies are based on surveys, interviews, and artifact examination $[2,4,6,14]$ which are sometimes combined with in-office or in-home observation $[5,9,13]$, our own experience from the first part of the study suggested the need for complementary methods. In study part 2 (conducted in the first half of 2013), the Day-Reconstruction Method [12] was applied.

In this section, a brief overview of the first part of the study is given and implications for the second part are discussed. The main part of this section is dedicated to the presentation of study part 2 .

\section{Study Part 1: exploratory interviews}

While many studies focus on homogeneous groups of participants (e.g., [2, 4, 6, 7, 19, 21, 23, 24]) we interviewed a variety of people with different backgrounds and realms of life because we were interested in getting a small insight into everyday calendar practices and their dynamics. The interviews were conducted with thirteen students from different fields and five professionals. We assumed that the participants had different experiences in using calendars. The professionals have developed their calendar work and routines over many years and have experienced the introduction of digital calendar tools. The student participants can (or could) choose between all sorts of digital and paper calendars when the need develops (or developed). The participants were recruited from the university of the authors and the surrounding community. Participation was voluntary and no incentive was offered. The demographics of the participants were as follows.

- The students were 19 to 30 years of age (eight male, five female), with six domestic students (German) and seven foreign (not German) students from six countries, of which eleven were graduate students, two PhD students. Two of the students were married and two had one child.

- The professionals were aged from 40 to 50 years (four female, one male, all German), and all were married and with children.

Interviews were open-ended semi-structured with questions similar to those in $[6,14]$ and ranged from 20 to 60 minutes. Informed consent was obtained from all participants for use of their anonymized data. All interviews were audio-taped and transcribed. The analysis was guided by the concepts and findings of previous research as examined in the related work section. The following codes are used below:

WP working participant,

SP student participant.

\section{Results from the interviews}

Calendar artifact collections: All participants own a mobile phone and employ several tools for their calendar work. The WPs have between two and four calendars, but only two SPs have more than one. All participants use paper notes in one form or another. For example, one SP claimed to use sticky notes for short term activities and alarms for long term tasks. Only one SP mentioned electronic notes. Two of the five WPs and six of the thirteen SPs use electronic reminders, but three of the SPs seldom use them. Eight SPs (but no WP) mentioned being reminded of birthdays on Facebook. Six SPs reported printing out their lecture schedules.

Electronic and paper calendars: Four SPs use an electronic calendar, seven a paper calendar, one has both, and one SP mostly relies on paper notes. From the seven SPs with a paper calendar, six use mass-produced exemplars and one SP creates his own calendar every month. Only two SPs explicitly mentioned the university's calendar and scheduling systems. Four of the five WPs have one or more paper calendar and all use one or more GCSs.

Personal and shared calendars: Three of the five WPs manage a private calendar in a GCS and three share a paper wall calendar with their family. One of the four SPs with an electronic calendar shares it with his friends (Fig. 2, right). He and one of the WPs were the only ones who reported using automatic synchronization. One SP mentioned a shared cleaning schedule in their dormitory.

Routines in calendar work: We asked participants about the routines they developed in their calendar work and about the interplay between their memory, the artifacts in use, and the environment. Most, but not all, participants 


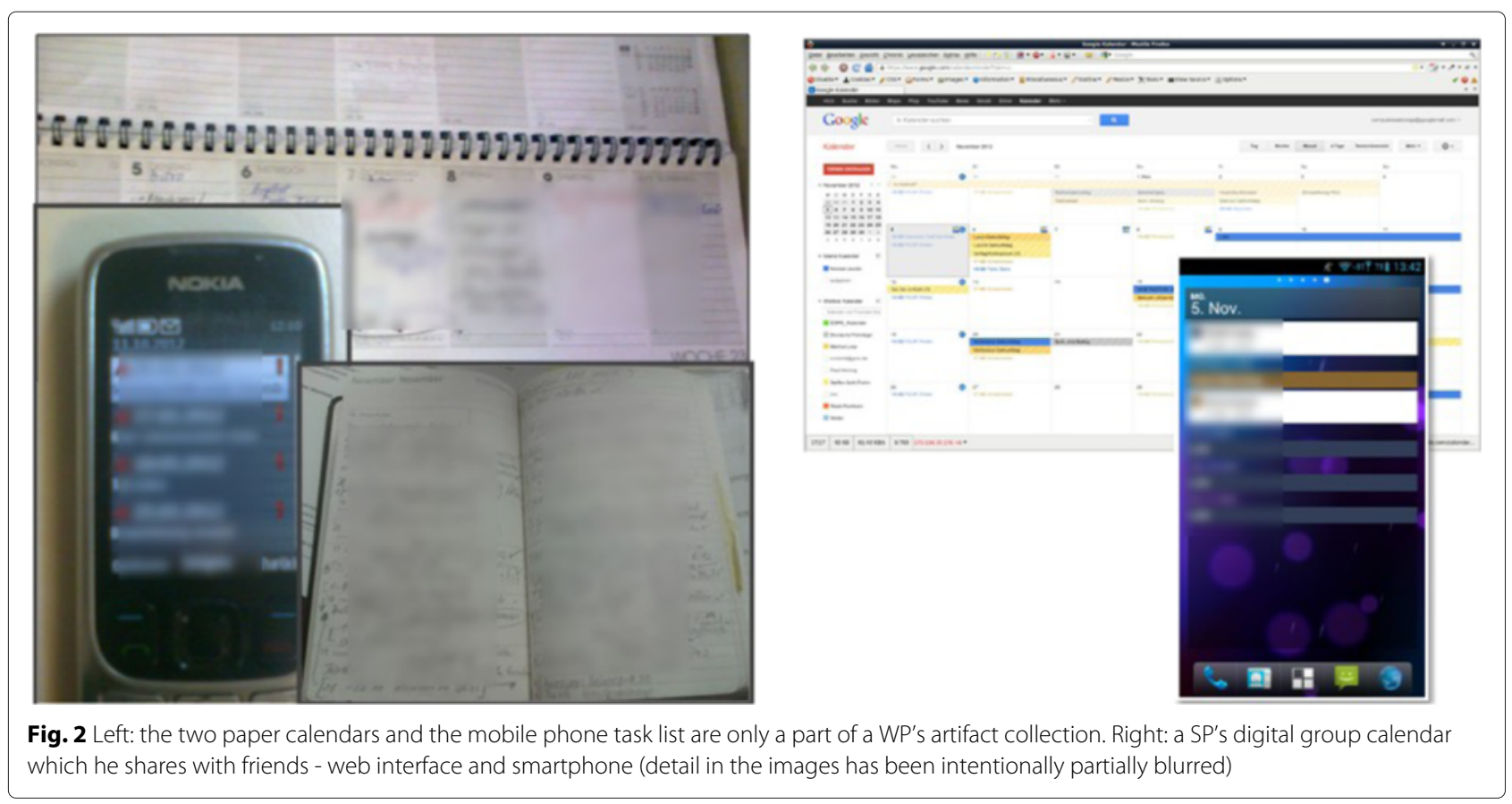

stated they read their calendar artifacts several times per day. Many SPs mentioned paper notes on their desk and most WPs keep their calendars open at work, regardless of whether they are paper or digital. Three of the five WPs write down relevant entries from electronic group calendars into a personal calendar (paper in two cases). The family calendar is located in the kitchen in all three cases mentioned above.

The importance of handwriting in their calendar work was emphasized by two SPs: I have this impression that if I don't write something down I would forget it. Only one of the SPs mentioned that he regularly 'uses' other people as reminders. The interviews revealed that participants develop and exploit additional mechanisms such as multiple paper notes in different places to be certain of triggering particular actions, which were often referred to as 'important appointments'. One of the PhD students tries to cope with important deadlines by creating multiple reminders within an interval of, say, 30 days. As another example, one SP reported his habit of putting paper notes in his trouser pockets if he really wants to be reminded to do something because they 'feel different' in the pocket than his mobile phone.

However, many participants claimed to still use their memory a lot. For example, a student said about familyrelated events: We talk regularly, so I can't forget it. Half of the students who, at the beginning of a semester, print out lecture schedules said that they have them memorized after two or three weeks. One SP employs alerts for lectures, but most other participants do not use external reminders for regular events.
(Potential) changes in calendar work: Some SPs reported that the need for a calendar has only developed recently. A student from Syria pointed out: In Germany, one always has to arrange long-term appointments, even with friends. This doesn't happen in Syria. Another student said that he did not need a calendar for a long time but could manage everything with his memory, some paper notes and reminders on his mobile phone. Now, he increasingly uses his paper calendar as well.

Although some of the participants mentioned occasional failures in calendar work all but one participant reported no urgent needs to fundamentally change their current calendar practices and they wished, at most, some 'adaptations' of their artifact collections. For example, one student wanted to have an additional monthly calendar in her room. However, in the past, two SPs tried to use digital calendars but returned to paper. One did not check the phone regularly or it was sometimes in the silent mode. The other said: I feel being always on the phone could be impolite... When I write a note in my [paper] calendar, it is obvious I'm not on Facebook or chatting. One WP described past failures in synchronizing between his paper and electronic calendars and his transition to electronic artifacts ten years ago. One student changed from paper to digital calendars with his new smartphone (Fig. 2, right). He describes himself as someone who loves technology. Some participants said that they perhaps would use an electronic calendar if they had a smartphone but they would not buy a new phone just for a more usable calendar than that available on their current mobile phone. 


\section{Discussion and implications for study part 2}

The interviews confirm that people use a variety of calendar artifacts in diverse ways and that there is no clear dominance of digital calendars over paper artifacts or vice versa (Blandford and Green [6], Tungare et al. [14]). The results indicate an increased and more systematic use of calendar artifacts by the working participants who reported higher demands of coordination and awareness in and across different realms of life (e.g., family, work) than most of the students. In particular, the working participants reported a more intense use of group calendars and of realm-specific calendars.

Many students had just started to develop a need for more external support in their calendar work. Although they grew up with computers, social networks and mobile phones and have easy access to digital calendar tools we could neither observe a general tendency towards a digital all-in-one tool nor that the younger participants have a significant higher preference of digital tools than the older participants. For example, eight of the thirteen student participants mentioned using Facebook for birthday reminders but only five students had electronic calendars and several students emphasized that they would not buy a new mobile phone just for a digital calendar. This suggests that the reasons for the use and the non-use of calendar tools are manifold and have to be understood at different levels. For example, the social pressure to apply certain calendar tools may vary in different contexts. The presence of a communication tool or a social network may make it a 'ready-to-hand tool' for some parts of a person's calendar work as well, but may even increase the complexity of his or her calendar artifact collection.

The results also suggest that the inconveniences and failures in the participants' calendar work need to pass a certain 'threshold' to encourage them to rethink and fundamentally change their practices, for example, by replacing current tools with new ones. For the participants, the maintenance of calendars is only a secondary task in most situations and this may impede their appropriation of new artifacts by developing corresponding usage routines. Previous studies and our exploratory interviews show that designers of (digital) calendar tools need to acknowledge the diversity and dynamics of calendar work and calendar artifact collections. The second part of the study presented in the next subsection aims to better understand these processes.

A well-known limitation of interviews is that interviewees 'rationalize' their behavior. It was easy for the participants to express opinions about certain tools and features (e.g., synchronization is easier with digital tools, or: I don't use this reminder function because I don't want to let the machine control what I have to do), but it was more difficult to list all employed artifacts. In addition, the interviews and the artifact examination convey a rather static view on calendar work, which is so ubiquitous that it is hard to observe directly if it is not restricted to certain domains. A method is needed that allows us to capture and analyze moments of the participants' calendar work and that supports their own reflection. In study part 2, the Day Reconstruction Method was applied.

\section{Study Part 2: follow-up study}

Calendar work of individuals is often described as idiosyncratic and diverse. This is supported by the unique calendar artifact collections that could be observed in previous studies and in the exploratory interviews. However, people's calendar work is also determined by mass-produced calendars, prevalent communication tools, group practices and the like. The objective of part 2 of the study was to get a deeper understanding of, and a less static view on, calendar artifact use. The concept of (personal) artifact ecology which is briefly described below guided the second part of the presented study.

\section{Theoretical basis}

Two different, but equally relevant perspectives on artifact ecologies are to be found in the literature. Bødker and Klokmose [28] and Jung et al. [29] consider personal artifact ecologies, whereas a more global view on artifact ecologies and artifact evolution is discussed by Kirsh [11] and Krippendorff [30].

Jung et al. [29] restrict themselves to the examination of interactive artifacts and emphasize that "the interconnected use of multiple interactive artifacts needs to be investigated [by researchers and designers] to fully understand their meanings and values in human life beyond technical and functional influences". The authors define a personal ecology of interactive artifacts as a set of all physical artifacts with some level of digital interactivity that a person owns, has access to, and uses. Artifact ecologies are described by ecology maps that visualize the relationships between the artifacts. Bødker and Klokmose [28] criticize this rather static image and point out that personal artifact collections are dynamic and evolving. In their study, they investigate how the appropriation of a new multi-purpose device influences, and is influenced by, the participants' existing artifact ecology. Appropriation, in this context, refers to the way people reshape the intended use of technological artifacts to get a sense of ownership and to achieve their purposes. In such processes, which are always embedded in social ones, a person learns to relate features of an artifact to the collection of currently used means and to his or her own needs and skills. The artifact starts to provide action possibilities or affordances [31]. "Learning to operate develops as does context of use" [28]. 
Kirsh [11] takes a more global approach. According to him, each artifact ecology is characterized by five elements that mutually constrain each other: 1) artifact species, 2) artifact systems or collections, 3) user groups who select and keep artifacts, 4) practices that users have evolved to work with artifacts and accomplish tasks, and 5) task environments. For Kirsh, artifact ecologies are systems of interdependent artifactual niches which "jointly determine how artifacts are used, when they are used, for what, by whom and how effectively". These systems are never stable because artifacts, tasks, practices, and users constantly interact and co-evolve. Krippendorff [30] defines an ecology of artifacts as the net effect of numerous personal decisions concerning the deployment of technology. He distinguishes cooperative, competitive, and independent interactions between two artifact species. Both authors provide explanations for effects of mass production (and advertisement) on individual behavior and vice versa. For example, the greater the prevalence of an artifact is the better users think it is compared with similar artifacts [11].

\section{Assumptions and research questions}

In study part 2, the unit of analysis is still the individual person, but it is assumed that a personal calendar artifact ecology unfolds and develops within and across the (calendar) practices of the different activity systems the person has been, or is, involved in. In the context of this article, activity systems are understood as durable systems that have been established by groups of people. They are driven by communal motives and reproduce themselves by corresponding practices (e.g., by timely rhythms) [32]. On the one hand, an individual's ecology of calendar artifacts is shaped by the various calendar practices of the activity systems he or she is engaged with and by his or her boundary work to move between the different systems. On the other hand, calendar artifact ecologies of activity systems are shaped by individual action and underlying values and motives. Kaptelinin and Nardi [33] point out that "[e]ven though human motivation is profoundly influenced by culture and society, each individual has her own hierarchy of motives". Therefore, personal ecologies of calendar artifacts can also shed light on possible net effects on a more global level. The following research questions were developed.

RQ1: Which forms of personal ecologies of calendar artifacts can currently be observed?

RQ2: How have these ecologies evolved? For example, what factors have influenced the selection and introduction of specific calendar artifacts and how did their appropriation influence current practices?

RQ3: What are the possible net effects of personal calendar artifact ecologies?

\section{Participants and method}

To answer the research questions, the focus of the second part of the study was on people with longtime experience in using calendar artifacts to meet the demands of coordination and awareness in their multiple activities. We analyzed personal calendar artifact ecologies of six participants (aged from 40 to 60 years) spanning a loose network through partly overlapping activities to emphasize their integration in different activity systems. Although they have comparable cultural and educational backgrounds their specific situations are surprisingly diverse and, in the context of this study, their network can be considered as a representative sample (Fig. 3). Table 1 gives an overview of the participants (Bea and Ben have already participated in study part 1). At the beginning of the study, informed consent was obtained from each participant for use of their anonymized data.

We aimed to collect data about the participants' actual calendar work and about its dynamics in the most unobtrusive manner possible and without introducing new calendar artifacts into their artifact ecologies. The DayReconstruction Method by Kahneman et al. [12] was applied because it facilitates off-line capturing of rich qualitative data. The method starts with an introductory interview. Then, participants are asked to reconstruct their previous day in a diary and as a sequence of episodes with a name, beginning and end, comments. To mitigate retrospective reporting biases, they later use these confidential notes when answering a series of questions from the researchers. A further advantage of the method is that it can easily be adapted to specific research contexts [12]. The adaptability of the method has been demonstrated by Karapanos et al. [34] in a study on user experience where participants created - over a period of four weeks and on the basis of their confidential diaries - daily experience narratives about the use of a new interactive device.

Participants in our study reconstructed and reflected upon their artifact use over a period of two weeks. At the end of each day, they created both the today's diary and a protocol of the previous day containing information about arrangements, calendar entries and notes they (or others) made for their episodes of that day, for other people's actions they wanted to be aware of, and for future plans. The participants used their diaries and calendar artifacts to complete the protocols. They could choose between paper or digital templates and were encouraged to add free comments. Interviews were conducted before and after the protocol phase. The second interview was informed by the protocols and allowed the participants' auto-confrontation [35] to reveal cognitive processes underlying their calendar work and to generate reflection about the current state and the dynamics of their calendar ecologies. 


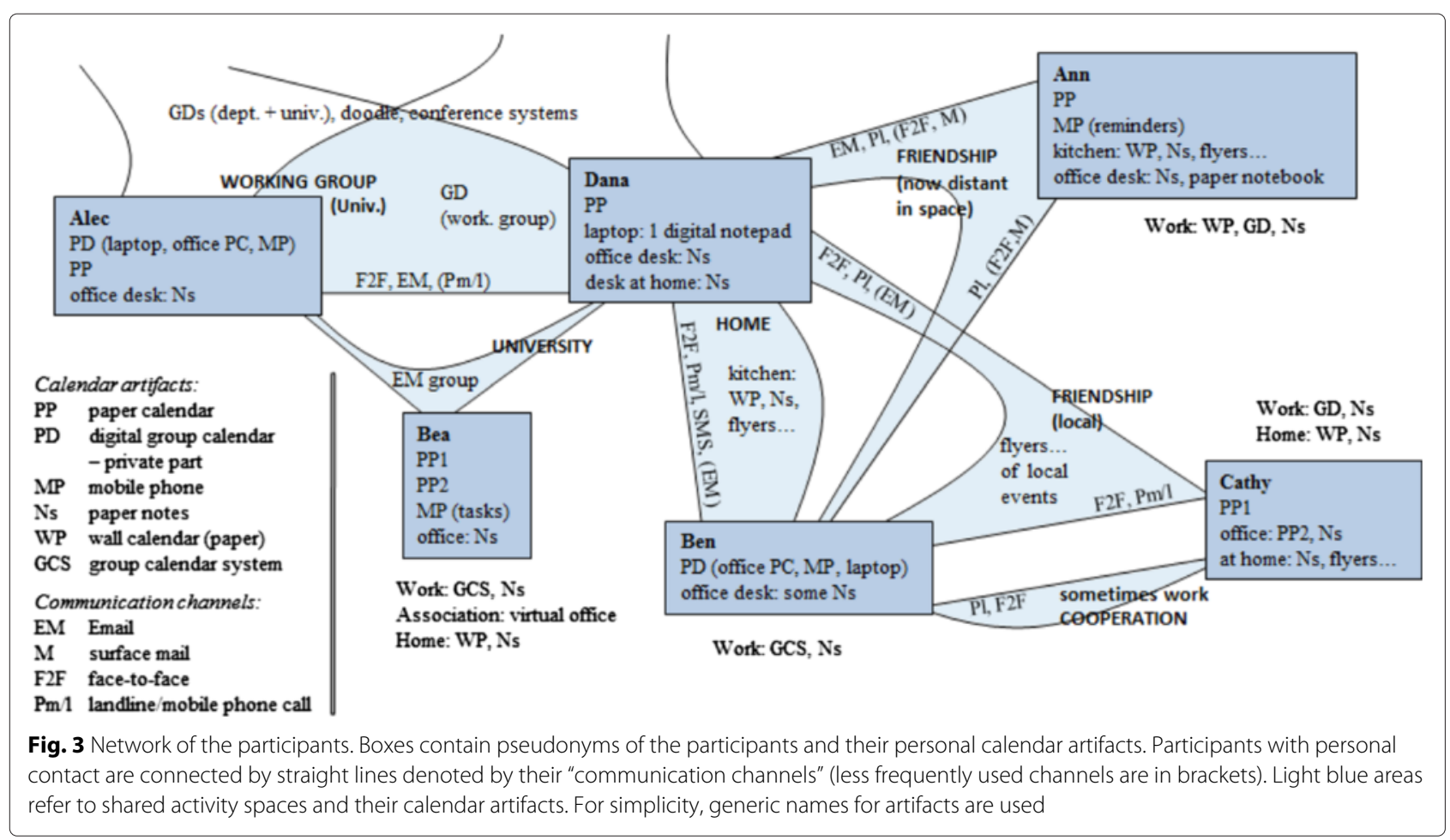

\section{Limitations}

The number of participants is low. The traced period of time is short and may not be typical. However, this was discussed in the interviews. Accuracy is still a shortcoming of the Day-Reconstruction Method. We could see, e.g., that Ben's entries into the kitchen calendar on Sunday (Fig. 6) were mentioned in Dana's protocol but not in Ben's. During the protocol phase,

Table 1 Overview of the participants

\begin{tabular}{|c|c|c|c|}
\hline Pseudonym & Family & Work & $\begin{array}{l}\text { Leisure } \\
\text { activities }\end{array}$ \\
\hline Ann & single & $\begin{array}{l}\text { technical project } \\
\text { coordinator }\end{array}$ & $\begin{array}{l}\text { choir, } \\
\text { music }\end{array}$ \\
\hline Bea & $\begin{array}{l}\text { married, two } \\
\text { young children }\end{array}$ & $\begin{array}{l}\text { coordinator } \\
\text { international } \\
\text { exchange } \\
\text { activities at } \\
\text { university } \\
\text { (half-time), artist }\end{array}$ & $\begin{array}{l}\text { art and } \\
\text { culture } \\
\text { association }\end{array}$ \\
\hline Cathy & $\begin{array}{l}\text { divorced, two } \\
\text { teenage children }\end{array}$ & $\begin{array}{l}\text { traffic planner in } \\
\text { municipal office }\end{array}$ & sport \\
\hline Dana & $\begin{array}{l}\text { married, grown-up } \\
\text { and teenage child }\end{array}$ & $\begin{array}{l}\text { assistant professor } \\
\text { (university) }\end{array}$ & $\begin{array}{l}\text { jewelery } \\
\text { design } \\
\text { course }\end{array}$ \\
\hline Alec & $\begin{array}{l}\text { long distance } \\
\text { relationship, two } \\
\text { grown-up children }\end{array}$ & $\begin{array}{l}\text { professor } \\
\text { (university) }\end{array}$ & \\
\hline Ben & $\begin{array}{l}\text { married with } \\
\text { Dana, two children }\end{array}$ & $\begin{array}{l}\text { department head } \\
\text { (engineering } \\
\text { company) }\end{array}$ & $\begin{array}{l}\text { music } \\
\text { band }\end{array}$ \\
\hline
\end{tabular}

the participants could already reflect upon and change their behavior although that typically requires a longer time.

\section{Data collection and analysis}

Interviews were audio-taped and transcribed. A first analysis of the protocols and pictures, which were collected and captured in digital form, informed the second interviews. The analysis of collected data was guided by the theoretical basis and by elicited themes described earlier in the paper. Diagrams have been created from protocols that give a schematic temporal overview of the participants' combined use of communication and calendar artifacts to manage their activities at work, with family and friends, and with acquaintances (also including doctor appointments and the like, see Fig. 4, Fig. 6).

\section{Results of study part 2}

Not surprisingly, all participants use a variety of paper and digital calendar artifacts, among them group calendar tools, stationary and portable tools. Figure 3 gives an overview of the participants and their calendar artifact collections. It also sketches their shared activity systems with shared calendar tools and expected communication means. In the figure, generic names for calendar artifacts are used and a reader may get the impression that the participants have quite similar calendar practices. In the first part of the results section, some more details about the participants' ecologies of calendar artifacts are given in narrative form to convey their diversity. Then, observed 


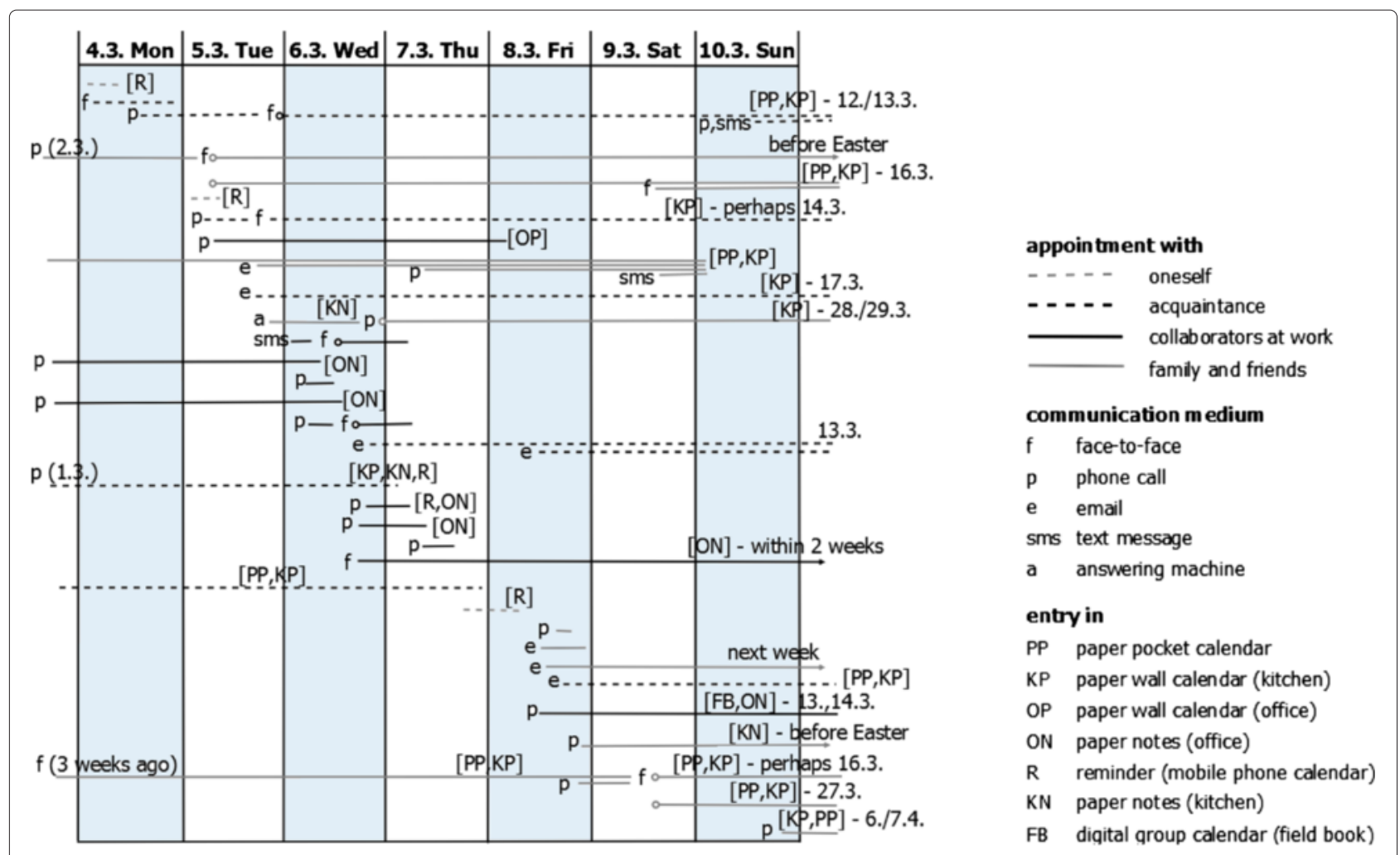

Fig. 4 Ann's calendar work in the first week

appropriation processes and changes in calendar work are described.

\section{RQ1: Which forms of individual ecologies of calendar artifacts can currently be observed?}

Ann works in an ecological long-term research project with 40 partners. She has to coordinate the local fieldwork with farmers and forest rangers. Every second day, she consults the project GCS (called FieldBook). In their local office, Ann and her three male colleagues share a paper 'family planner'. Figure 4 shows the recorded tool use of a quiet week at work where Ann created six paper notes for her office desk, five of them after a phone call. Her central calendar for private issues is the paper wall calendar in her kitchen. Some entries, especially plans for weekends and work-related appointments outside working time, are copied in the paper pocket calendar for use away from home. Ann uses her old mobile phone for reminders if, in a certain situation, no paper is available or if it is important for her not to forget to do something. Figure 4 shows examples of her 'lunch-break reminders' at work helping her to think of necessary private errands in town (see also Fig. 5, left). She had an electronic reminder, an entry in the kitchen calendar, and a paper note for the hairdresser appointment on Thursday morning. She knows she would easily forget it and she did not want to disappoint her hairdresser. For almost 20 years, Ann and Dana have had long phone calls which they recently started to schedule by email. Dana sent an email on Friday and they had a call in the evening (Fig. 4).

Dana had no reminder for this email, but her notes and pocket calendar often contain items such as "call Uwe" or just the name of a person to finally trigger her (or another family member) to contact this person. The tension between short-term and long-term tasks in Dana's work is well described in [6]. She deliberately distinguishes between tasks to do at the university and tasks she can better complete at home. Figure 5 (right) illustrates that her use of notes supports this separation and also a separation of work-related and other activities at home. In Dana's case, most appointments are made by email or face-toface, or they are specified by a schedule. Dana's pocket calendar almost exclusively contains work-related items and she 'misuses' the kitchen wall calendar for her own dental appointments although this is not interesting for the family. On February 27, 2013, Dana reminds her group head of a meeting and they agree on March 7. She makes a note in her paper calendar and later gets an email invitation from the GCS which she confirms again. She knows now that the appointment is also in Alec's calendar. 


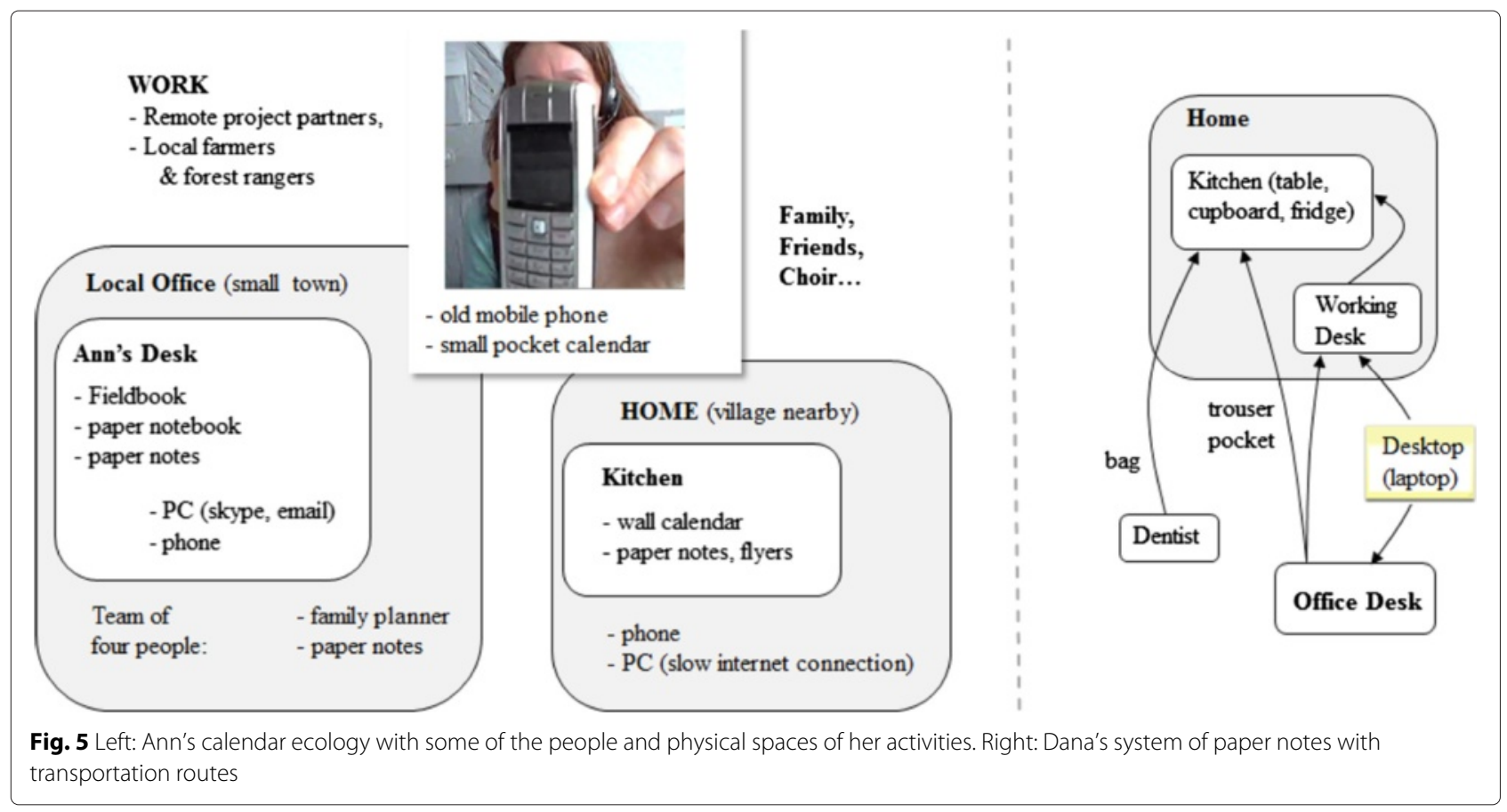

Alec's protocol shows an international working group meeting and oral examinations as scheduled events, and evening phone calls with the family without reminders. Some other meetings were arranged by Doodle - a tool he learned to appreciate. Sometimes, he asks his secretary to arrange appointments and to write in his on-line calendar. He still has a paper calendar, which mainly serves as a notebook and as a source for entries into the electronic calendar (never vice versa). There is no shared calendar in Alec's home. On February 17, 2013 (Sunday), he and his partner compare their calendars for holiday planning.

Ben - in contrast to Alec - automatically synchronizes his Lotus Notes calendar instances every evening and heavily employs reminders (see Fig. 6). In his work domain, many clients and other remote collaborators expect to be contacted by phone, but it is sometimes difficult to get them on the phone. He learned to use reminders for repeating calls to keep his head clear for other tasks. At the end of the protocol phase, he also had a morning reminder to water tomato seedlings at home. The GCS helps Ben to be informed about 'his' engineers' external appointments and to respond, if necessary, to phone calls or other requests concerning their projects. Over the last ten years, he learned to make use of most of the calendar entry types, sometimes in unintended ways. For example, he uses tasks to be aware of vacations of colleagues. He has a weekly appointment for Thursday evening - not because he would forget to drive to his mother for weekend shopping but because he might not be aware of it in work meetings when arranging new appointments (Fig. 7). Ben's and Dana's children are grown up now. The paper class schedules disappeared from the kitchen (Fig. 9) and entries in the kitchen wall calendar rather support their awareness of the children's activities, but they can remember very well a time...

Bea is experiencing this now. Her family and private calendars are full of details of who fetches and drops off the children. Bea has to bring together many different activities (Table 1) and uses separate calendars for managing it (Fig. 2). She also uses the task list of her mobile phone to be reminded of art projects two weeks in advance. Every Sunday night, she and her husband open all their calendars (paper and electronic) to plan for the next week, to share family tasks and to 'synchronize' the calendars. The kitchen wall calendar plays a central role in this process. Her protocol also shows an active use of the GCS by all colleagues at the university.

Cathy Cathy works as a traffic planner in a municipal office. In her work, it is important to be aware of the current state of municipal projects and of regulations, deadlines and decisions made in the municipal office. She considers the 'heaps' (of paper notes and protocols) on her work desk an important part of her calendar artifact collection. Here is a part of a comment from her protocol that not only shows her handling of calendar artifacts (see also Fig. 8) but also her reflection on the tension between planned and spontaneous actions: I think, I ignore (for whatever reason) my original plans, especially in private areas of my life. No memo can help here! Nevertheless I do 


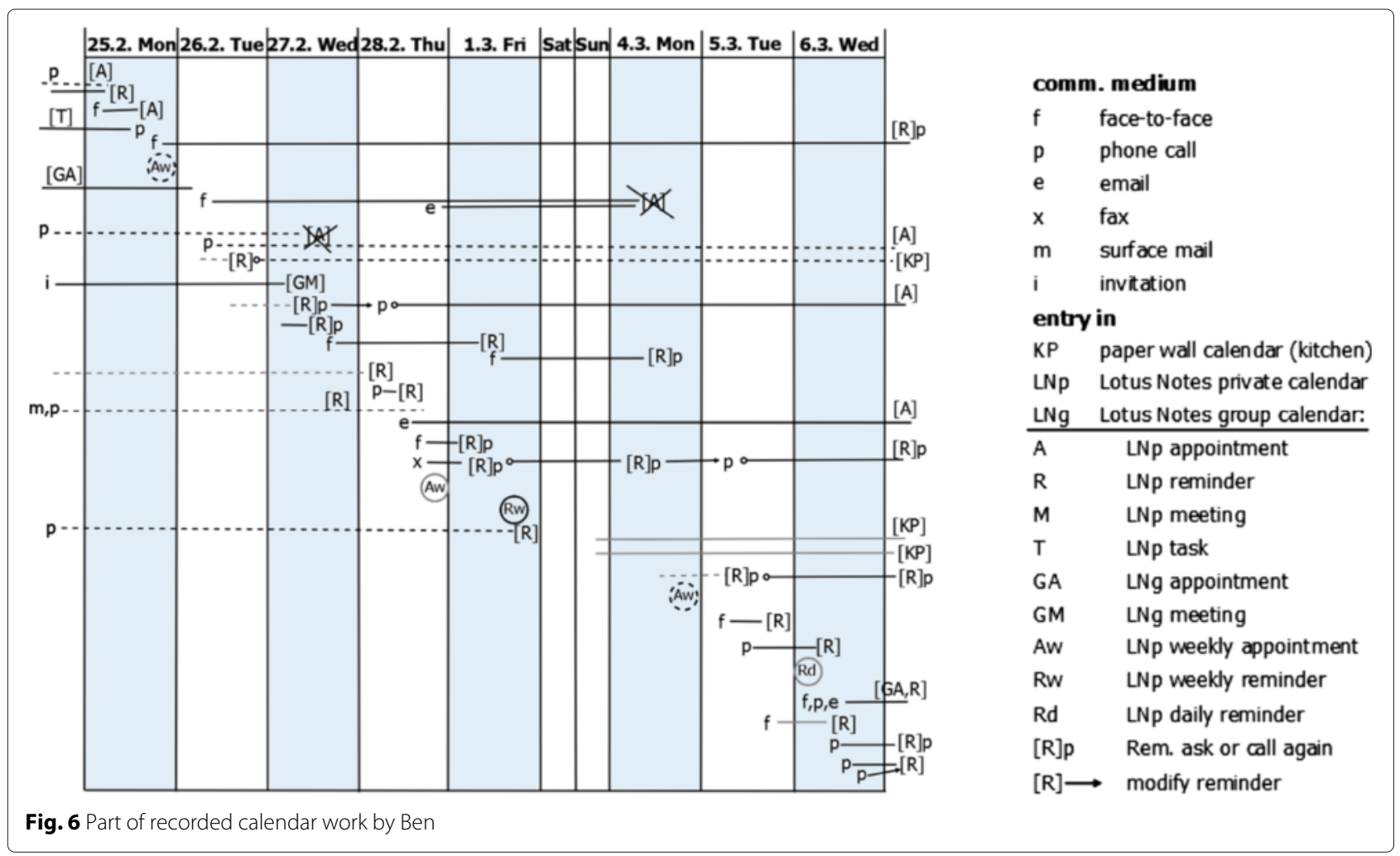

something else instead. I also discovered that I have notes of different types. There are portable and non-portable notes, leaflets, which I sometimes put on a pile together with cinema and theater programs... Some notes make the leap to the pin board, some even to the kitchen wall calendar... Then, fixed appointments which are not really fixed: my sport between 11:00 and 13:00, which I registered for Monday and Wednesday in Outlook [GCS at work], but I actually do it when I feel like it, anytime in the week, and some weeks I don't do it at all. Then sauna: I have the opening hours on my pin board and go: sometimes Wednesday, sometimes Monday... I have no notes for it; I have it in my mind.

RQ2: How have these ecologies evolved? What factors have influenced the selection and introduction of specific calendar artifacts and how did their appropriation influence the calendar artifact ecologies of the participants? The participants described minor and major changes in their calendar work, which were mainly triggered by changing demands within an activity system and adjustments of their boundary work between multiple activities,

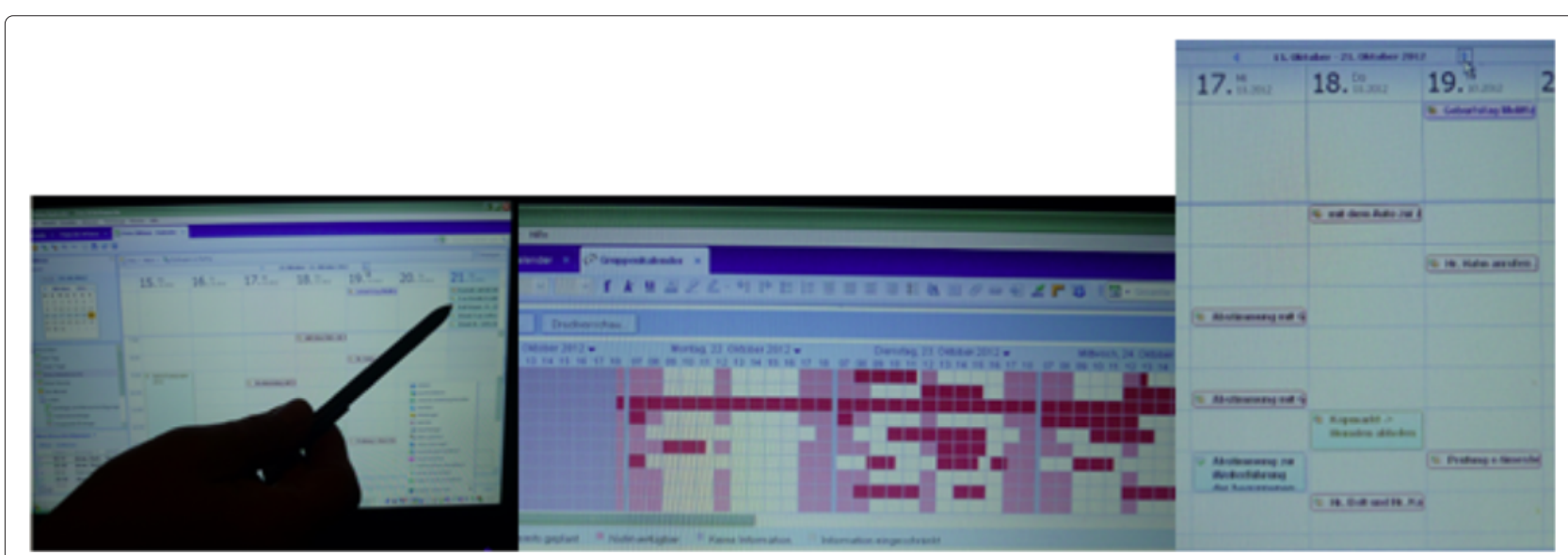

Fig. 7 In Ben's calendar ecology, the digital calendar has a central role. It is accessed through three devices 

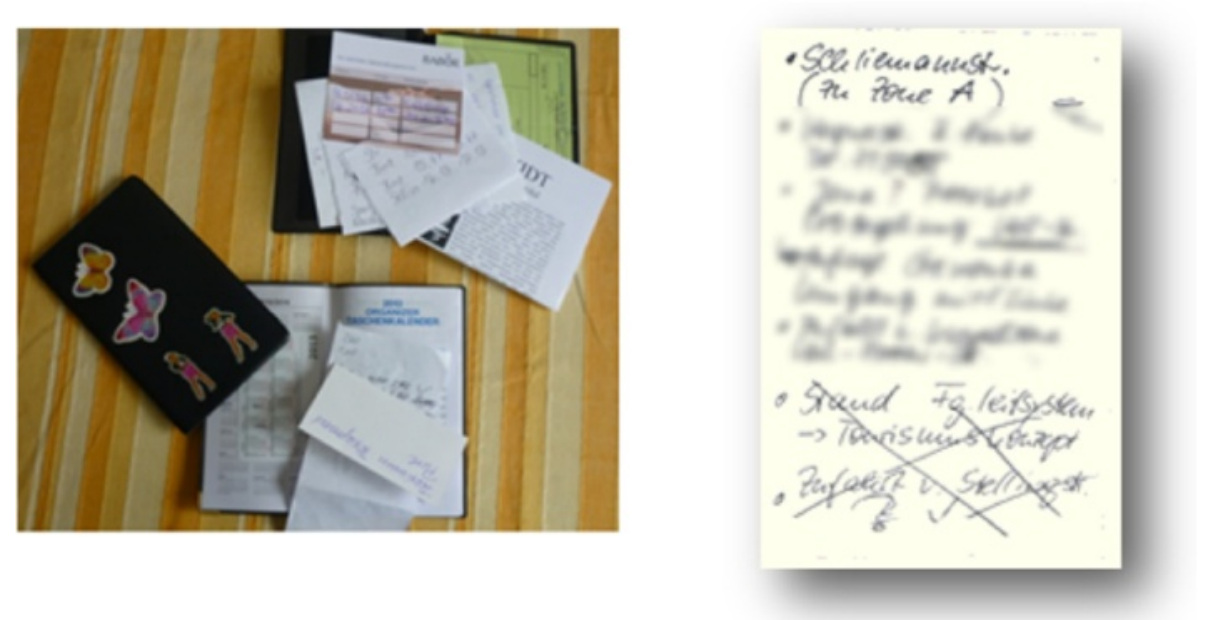

Fig. 8 Left: Portable calendar artifacts in Cathy's bag. Right: Example of a stationary paper note on her work desk which mainly helps her to be reminded of her 'awareness tasks' (detail in the images has been intentionally partially blurred)

by unacceptable failures of their calendar work due to deficiencies of the currently used artifact collection, and by the willingness to explore and/or the request to use new artifacts. For example, Dana added digital notes to her system of paper notes (Fig. 5, right) to better cope with the increasing demands of short-term tasks by decreasing the separation between 'office desk tasks' and 'home desk tasks' that was mentioned above. However, she soon reduced her use of digital notepads to one notepad only. Alec reported that he got rid of his schedule of garbage pick up times because he sometimes failed to maintain it. Now, he just follows his neighbor when putting out the bins. Ann started to use paper pocket calendars in her early forties. Her first exemplar was a Christmas present and she was willing to explore its usefulness because it was so beautiful. However, the wall calendar in her kitchen is still her central calendar for private issues and she reported that although she now always buys paper pocket calendars, they are still less frequently used towards the end of a year.

All participants experienced in their professional life the introduction of GCSs. In Ben's case, Lotus Notes was introduced in his company 15 years ago and the engineers were encouraged to use it. He started to use the digital calendar on the PC in his office but still used his paper calendar for meetings outside. When he missed an important appointment due to bad synchronization work he decided to buy a PDA and to throw away his paper calendar. This bad experience may also explain his routine activation of the automatic synchronization function. His experiences with the GCSs later influenced its use by his team. The above mentioned GCS of Dana and Alec is only used in the research group and has not replaced their paper calendars. It has changed the role of Alec's paper calendar, but not that of Dana's. She explained that, in her case, there is no urgent need to make her other appointments visible to the group through the GCS. Additionally, her mobile phone is often switched off while her paper calendar is always ready to hand. In Ann's case, the GCS of all project partners (FieldBook) needed to be complemented by other artifacts. She and her local colleagues soon asked the remote researchers for an additional email or phone call to confirm their reservations and needs in FieldBook (e.g., Fig. 4, Friday). She reported that it was embarrassing for her to make appointments with farmers, and then, some researchers did not attend.

Furthermore, a diverse use of 'family' wall calendars could be observed. For Bea it is a central instrument in 'synchronizing' her and her husbands' calendars. Ann and her local colleagues use it as group calendar in their office. Ann also uses a family planner in her kitchen as her main private calendar. Dana and Ben used their kitchen wall calendar in a similar way like Bea but now mainly for planning weekend visits or events as well as for being aware of each other's business trips and some of their children's activities. Additionally, Dana uses it for some private entries.

The participants described the network of activity systems they are involved in as more complex than 'Home' and 'Work' (a distinction that was investigated by NippertEng [22]) and with more or less flexible boundaries. All participants used both integrating as well as segmentation strategies to mutually adjust their involvement in multiple activity systems. Stationary artifacts played an equally important role to portable ones. This becomes even obvious in Ben's description of his transition from a PDA to a smartphone. Although he started to use paper notes as temporary buffers for appointments (a strategy that was 
described in [23]) he also sees advantages in this lack of usability of his smartphone because it supports his effort not to check his work calendar during weekends.

\section{Discussion}

When asked whether she can imagine living without a calendar, Bea said that this would mean she had to be another person. Calendar artifacts are "tools that help people both do and be" [1]. This may explain why all participants were interested in the study although the protocol phase was demanding.

RQ1: The results deliver an insight into the unique personal calendar artifact ecologies that have been developed over time. Tomitsch et al. [15] state that a personal calendar represents an integrated all-in-one tool and the properties of paper support this functionality more efficiently than rigid digital calendars. The results of our study suggest, though, that there is rarely an 'all-in-one tool' (see, e.g., Fig. 3) and that the actual use of single calendar artifacts can neither be fully understood by their properties or offered functionality, nor by the consideration of tasks, user characteristics and tools in one activity system $[5,17]$. One has to consider its role in the overall calendar artifact ecology of a person which is shaped by the (temporal) structure of their web of activities, by selected artifacts and practices in the different groups, by their tasks and responsibilities, and by their relations to others and to themselves (including power relations and 'user characteristics'). The 'family' wall calendar is shown as one example of the diversity in reshaping the intended use of an artifact according to the users' needs.

It is also shown that the borderline between personal and shared calendars or between communication and calendar artifacts is blurred. What is Bea's personal calendar? One of her paper calendars, her notes on the office desk, or the task list in her mobile phone? Even Ben's personal digital calendar is not only for personal use. For example, he mentioned an incident where his boss asked him to make an entry in his calendar so that they cannot forget it?

RQ2: Kirsh [11] points out that artifact ecologies are never stable because artifacts, tasks, practices, and users constantly interact and co-evolve. This is even more true for personal calendar artifact ecologies which develop from the demands of a person's multiple activities. The results show instances of minor and major changes of calendar work and corresponding adaptation or extraction processes of existing tools and appropriation processes of new tools respectively. The development of multiple reminders is an example of a minor change of the artifact ecology to strengthen the trigger mechanism for action. An example for a major change is Ben's appropriation of the GCS offered by his company that first resulted in an expansion of his calendar artifact collection (paper calendar and GCS), and then in an artifact replacement (PDA instead of paper calendar). His use of the digital tool clearly contradicts the above mentioned claims made by Tomitsch et al. [15]. Generally, the appropriation of an artifact can result in an artifact expansion because it does not fully replace existing artifacts or it is complemented by other new tools, or it can result in a reduction of the artifact collection. Figure 10 illustrates effects of introducing calendar artifacts into, or extracting calendar artifacts from, personal collections.

Calendar artifacts can be introduced into or extracted from a personal ecology by social pressure or by power relations in a group. The increased use of Doodle by Alec may be explained by the characteristics of some of his meetings where one cannot expect the participation of all invited people. In addition, net effects as mentioned by Krippendorff [30] may have increased the prevalence of this tool even if it is not appropriate for each situation as reported by Alec.

Changes in calendar use are also caused by evolving activities and by changes as people age leading to changes in tasks and in coordination and awareness demands. An example is given in Fig. 9: children grow up and become independent from their parents. The example also demonstrates that simple calendar artifacts can coexist with more advanced ones. Calendar practices of individuals and groups are determined to a large extent by the temporal structure of their web of activities. For example, Ann and Bea have high scheduling demands in work activities and in their private life. Ann and Cathy sometimes find it a burden that they are expected - as single/divorced woman - to always have time for appointments. In contrast, rich rhythmic structures (a high degree of allocation) do not require much calendar work and individuals can more easily separate their artifacts for different groups and activities. In Dana's and Ben's family, there is no current need for an electronic family calendar. Ben's digital calendar co-exists with the paper artifacts at home and his 'synchronization efforts' are low. Baumer and Silberman [36] recommend avoiding a technological intervention (intended non-use) if there is "an equally viable low-tech or non-technological approach to the situation".

RQ3: The third research question concerns the net effects of personal calendar artifact ecologies. Our methodological approach alone cannot fully address this question, but we need to complement it with approaches studying calendar artifact ecologies from other than the personal perspective. However, the presented study puts an individual's calendar work into a larger context. Individuals move within and between different activity systems or realms with their own calendar systems. The results indicate how calendar practices and attitudes of individuals can influence, for example, the selection of calendar tools used in a group. This causes a change of the 


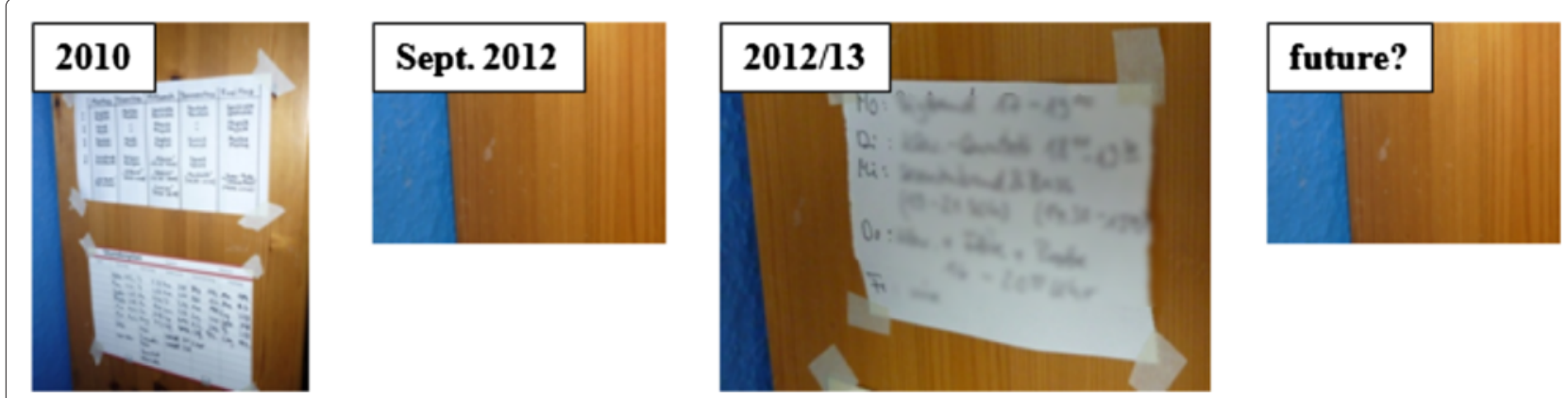

Fig. 9 Temporary failures in calendar work: class schedules of two teenagers (created by them) $\rightarrow$ nothing $\rightarrow$ schedule of the second child's leisure activities that Ben created after wondering where his daughter is (detail in the images has been intentionally partially blurred)

practices of all group members (see Fig. 10) and hence may influence the calendar work in their other activity systems. In other words, an individual's calendar work is shaped by and shapes the overall use of calendar artifacts. A deeper understanding of possible net effects of personal calendar artifact ecologies also requires a better understanding of deliberate non-use of certain calendar artifacts and of forms of technology resistance.

\section{Conclusions}

Personal use of calendar tools has to be understood in a broader context. The paper explored personal calendar artifact ecologies and their dynamics. It is demonstrated that appropriation processes of calendar artifacts are diverse and must be explained by an individual's unique situation. The results help designers understand that a successful design of calendar tools goes beyond the consideration of product usability and functionality and that they need to be aware of the effects of introducing new calendar tools to support the needs of their users more effectively. Sometimes a co-existence between lo-tech and hi-tech solutions is desirable, sometimes a new tool should reduce the existing calendar artifact collection, and sometimes a viable low-tech solution is to be preferred. Calendar tools should be flexible enough to be easily changed and integrated into existing artifact ecologies.

The potential of ecological perspectives for supporting the analysis and design of interactive artifacts is increasingly discussed in the field of HCI [37]. This paper contributes to the discussion by investigating calendar artifact ecologies from an individual's perspective. Future work includes the study from a more global perspective to better understand net effects of personal practices and to facilitate sustainable design of calendar tools. We utilized in our study the Day-Reconstruction Method to facilitate the participants' reconstruction of their calendar work. We believe that the application of this method to study personal artifact ecologies is promising, but requires further analysis.

In their study, Leshed and Sengers [1] come to the conclusion that many North Americans consider calendar artifacts as 'productivity tools' and thus reinforce the cultural emphasis of business. Our study design allowed participants and researchers to collaboratively reflect on the participants' 'time management' and underlying values. The results show the existing 'appointment culture'. However, they also show the importance of calendar

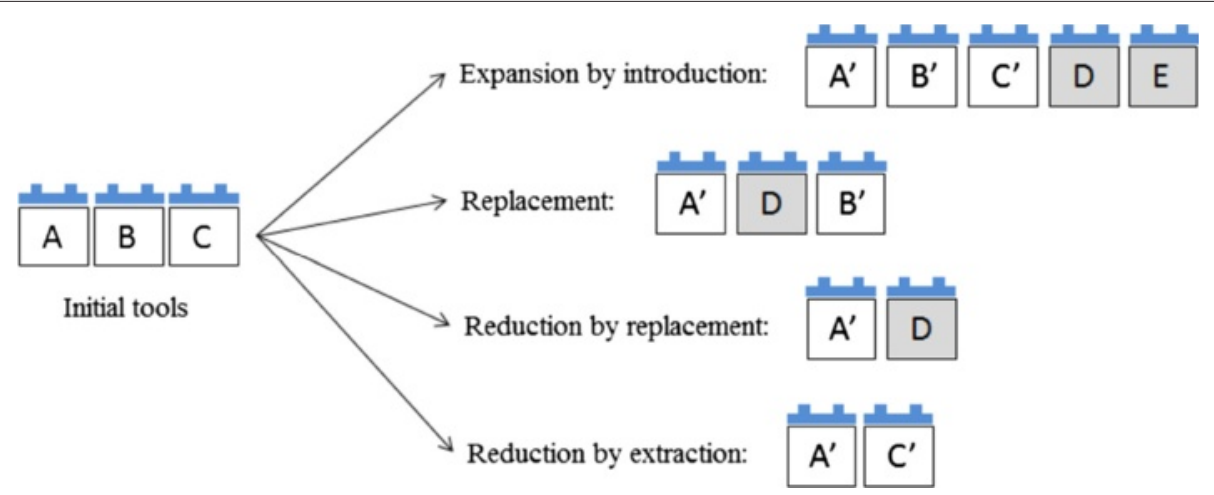

Fig. 10 Illustration of the dynamics in personal calendar artifact collections. The introduction of new tools (depicted by gray boxes) or extraction processes may also change the use of the other calendar artifacts in the collection (indicated by $A^{\prime}, B^{\prime}, C^{\prime}$ ) 
artifacts for maintaining work and personal relationships in the light of the travel and new technologies for communication.

\section{Competing interests}

The authors declare that they have no competing interests.

\section{Authors' contributions}

AD has formed the main research idea and the design of the study. LD conducted the interviews and the data collection activities. AD assisted these activities. Both authors analyzed and interpreted the data. AD drafted the manuscript. Both authors read and approved the final manuscript.

\section{Acknowledgements}

Our thanks go to the participants in the studies and to Fahimeh Shafaei for her support. Further, we thank Judy Bowen and the anonymous reviewers for their careful reading of our manuscript and their valuable suggestions.

\section{Received: 3 December 2014 Accepted: 5 July 2015}

Published online: 28 July 2015

\section{References}

1. Leshed, G, \& Sengers, P (2011). "I Lie to Myself That I Have Freedom in My Own Schedule": Productivity Tools and Experiences of Busyness, In Proceedings of the SIGCHI Conference on Human Factors in Computing Systems, CHI'11 (pp. 905-914). New York, NY, USA: ACM.

2. Kelley, JF, \& Chapanis, A (1982). How Professional Persons Keep Their Calendars: Implications for Computerization. Journal of Occupational Psychology, 55, 241-256.

3. Kincaid, CM, Dupont, PB, Kaye, AR (1985). Electronic Calendars in the Office: An Assessment of User Needs and Current Technology. ACM Trans. Inf. Syst., 3(1), 89-102.

4. Payne, SJ (1993). Understanding Calendar Use. Human-Computer Interaction, 8(2), 83-100

5. Palen, L (1999). Social, Individual and Technological Issues for Groupware Calendar Systems, In Proceedings of the SIGCHI Conference on Human Factors in Computing Systems, CHI'99 (pp. 17-24): ACM.

6. Blandford, A, \& Green, TRG (2001). Group and Individual Time Management Tools: What You Get is Not What You Need. Personal and Ubiquitous Computing, 5(4), 213-230.

7. Sell, A (2002). Mobile Managerial Time Management: A Study on High-Ranking Managers' Time Management Needs and Attitudes, In Proceedings of Decision Making and Decision Support in the Internet Age (pp. 526-536)

8. Sell, A, \& Walden, P (2006). Mobile Digital Calendars: An Interview Study, In 39th Hawaii International International Conference on Systems Science HICSS-39: IEEE Computer Society.

9. Crabtree, A, Hemmings, T, Rodden, T, Mariani, JA (2003). Proceedings of the 2003 European Conference on Computer Supported Cooperative Work. ECSCW'03. In K Kuutti, EH Karsten, G Fitzpatrick, P Dourish, K Schmidt (Eds.) (pp. 119-138). Netherlands: Springer.

10. Neustaedter, C, \& Bernheim Brush, AJ (2006). "LINC-ing" the Family: The Participatory Design of an Inkable Family Calendar, In Proceedings of the SIGCHI Conference on Human Factors in Computing Systems. CHI'06 (pp. 141-150). New York, NY, USA: ACM.

11. Kirsh, D (2010). The Cognitive Life of Things: Recasting Boundaries of the Mind. In L Malafouris \& C Renfrew (Eds.) (pp. 121-144). Cambridge: McDonald Institute for Archaeological Research.

12. Kahneman, D, Krueger, A.B, Schkade, D.A, Schwarz, N, Stone, AA (2004). A Survey Method for Characterizing Daily Life Experience: The Day Reconstruction Method. Science, 306(5702), 1776-1780.

13. Taylor, AS, \& Swan, L (2005). Artful Systems in the Home, In Proceedings of the SIGCHI Conference on Human Factors in Computing Systems. CHI' 05 (pp. 641-650). New York, NY, USA: ACM.

14. Tungare, M, Pérez-Quiñones, MA, Sams, A (2008). An Exploratory Study of Calendar Use. CoRR, abs/0809.3447.

15. Tomitsch, M, Grechenig, T, Wascher, P (2006). Personal and Private Calendar Interfaces Support Private Patterns: Diaries, Relations, Emotional
Expressions, In Proceedings of the 4th Nordic Conference on

Human-computer Interaction (pp. 401-404). New York, NY, USA: ACM.

16. Churchill, EF (2011). Making Time. Interactions, 18(3), 80-82.

17. van den Hooff, B (2004). Electronic coordination and collective action: use and effects of electronic calendaring and scheduling. Information \& Management, 42(1), 103-114.

18. Mosier, J, \& Tammaro, S (1997). When Are Group Scheduling Tools Useful? Computer Supported Cooperative Work (CSCW), 6(1), 53-70.

19. Salzman, M, \& Palen, L (2004). The Tools We Live By: A Description of Personal Support Media in Work Life. Computer Science Technical Reports CU-CS-981-04. http://scholar.colorado.edu/csci_techreports/918.

20. Crabtree, A, \& Rodden, T (2004). Domestic Routines and Design for the Home. Computer Supported Cooperative Work, 13(2), 191-220.

21. Brush, AJB, \& Turner, TC (2005). A Survey of Personal and Household Scheduling, In Proceedings of the 2005 International ACM SIGGROUP Conference on Supporting Group Work. GROUP'05 (pp. 330-331). New York, NY, USA: ACM.

22. Nippert-Eng, C (1996). Calendars and Keys: The Classification of 'Home' and 'Work'. Sociological Forum, 11(3), 563-582.

23. Starner, TE, Snoeck, CM, Wong, BA, McGuire, RM (2004). Use of Mobile Appointment Scheduling Devices, In CHI '04 Extended Abstracts on Human Factors in Computing Systems. CHIEA '04 (pp. 1501-1504). New York, NY, USA: ACM.

24. Taylor, AS, \& Swan, L (2004). List Making in the Home, In Proceedings of the 2004 ACM Conference on Computer Supported Cooperative Work. CSCW'04 (pp. 542-545). New York, NY, USA: ACM.

25. Zerubavel, E (1981). Hidden Rhythms: Schedules and Calendars in Social Life. Chicago: University of Chicago Press.

26. Dittmar, A, Kühn, R, Forbrig, P (2011). Coordination in Perioperative Systems: A Tacit View, In Proceedings of the 1st International Workshop on Engineering Interactive Computing Systems for Medicine and Health Care. co-located with EICS'11, ISSN 1613-0073.

27. Bardram, J (2000). Temporal Coordination - On Time and Coordination of Collaborative Activities at a Surgical Department. Computer Supported Cooperative Work, 9(2), 157-187.

28. Bødker, S, \& Klokmose, C.N (2012). Dynamics in artifact ecologies, In Nordic Conference on Human-Computer Interaction, NordiCHI'12, Copenhagen, Denmark, October 14-17, 2012 (pp. 448-457). New York, NY, USA: ACM.

29. Jung, H, Stolterman, E, Ryan, W, Thompson, T, Siegel, M (2008). Toward a Framework for Ecologies of Artifacts: How Are Digital Artifacts Interconnected Within a Personal Life? In Proceedings of the 5th Nordic Conference on Human-computer Interaction: Building Bridges. NordiCHI'08 (pp. 201-210). New York, NY, USA: ACM.

30. Krippendorff, K (2006). Semantic Turn: A New Foundation for Design. London, New York: Taylor\& Francis Group, CRC Press.

31. Bærentsen, K.B, \& Trettvik, J (2002). An Activity Theory Approach to Affordance, In Proceedings of the Second Nordic Conference on Human-computer Interaction. NordiCHI'02 (pp. 51-60). New York, NY, USA: ACM.

32. Engeström, Y (2000). Activity theory as a framework for analyzing and redesigning work. Ergonomics, 43(7), 960-974.

33. Kaptelinin, V, \& Nardi, B.A (2006). Acting with Technology: Activity Theory and Interaction Design. Boston: MIT Press.

34. Karapanos, E, Zimmerman, J, Forlizzi, J, Martens, J-B (2009). User Experience over Time: An Initial Framework, In Proceedings of the SIGCHI Conference on Human Factors in Computing Systems. CHI'09 (pp. 729-738). New York, NY, USA: ACM.

35. Mollo, V, \& Falzon, P (2004). Auto- and allo-confrontation as tools for reflective activities. Applied Ergonomics, 35(6), 531-540.

36. Baumer, EPS, \& Silberman, MS (2011). When the Implication is Not to Design (Technology), In Proceedings of the SIGCHI Conference on Human Factors in Computing Systems. CHI'11 (pp. 2271-2274). New York, NY, USA: ACM.

37. Blevis, E, Bødker, S, Flach, J, Forlizzi, J, Jung, H, Kaptelinin, V, Nardi, B, Rizzo, A (2015). Ecological perspectives in hci: Promise, problems, and potential, In Proceedings of the 33rd Annual ACM Conference Extended Abstracts on Human Factors in Computing Systems. CHIEA'15 (pp. 2401-2404). New York, NY, USA: ACM. 